\title{
Challenges Potential and Future of Internet of Things Integrated with Blockchain
}

\author{
Anubhav Kumar, Gaurav Jha, Lavanya Sharma, Sunil Kr. Khatri
}

\begin{abstract}
In the Internet of Things (IoT) idea, regular gadgets end up savvy and self-governing. As we are seeing idea behind this is transforming into a realism on account of achievement in innovation, but we still face challenges, especially in some field like security e.g., information dependability. Taking account, the upcoming advancement in the field of IoT, it seems very important to give trust in the field of enormous approaching data foundation. Blockchain has given us the new way to share our data with others. Building such a trust in discrete condition with is term as the key factor of blockchain in which we don't need any specialists is a hi-tech development that can possibly change numerous enterprises, the IoT among them. Troublesome advances, for example, IoT used enormous information and distributed to beat its restrictions, and we blockchain can be one of the accompanying ones. This paper centers around this correlation, explores difficulties in blockchain with IoT implementation, and reviews recent significant work so as to investigate about how the existing blockchain technology can change the way we work in IoT.
\end{abstract}

Index Terms: Anonymity, application, blockchain, decentralization, difficulties, innovation, Internet of things, security, smart environment, strength, trust.

\section{INTRODUCTION}

The internet of things (IoT) spread an idea which portrays the connection of regular devices with the internet but with the capability to recognize themselves as an individual and different device. This technology is closely related to the RFID "radio-frequency identification" for communication strategy, even it may absorb the other technologies like OR codes, wireless technologies or to some extend sensors technologies [1]. On the other hand, blockchain is an advanced record of transactions. Blockchain was originated from the concept where each block termed as record are connected together in a form of chain hence named as block-chain. There is multiple use of this technologies like cerographic form of money commonly known as bitcoin, and it is also used by banks to store their data in secure way. Most would agree in a couple of years' times, the physical world will be incredibly interconnected and rather smart. Be that as

it may, this additionally speaks to an issue, since in their

Revised Manuscript Received on July 5, 2019.

Anubhav Kumar, Amity Institute of Technology/ Amity University / Noida, India,

Gaurav Jha, Amity Institute of Technology/ Amity University / Noida, India.

Lavanya Sharma, Amity Institute of Technology/ Amity University / Noida, India.

Sunil Kr. Khatri, Amity Institute of Technology/ Amity University / Noida, India. present structure, IoT gadgets are astoundingly unreliable. IoT has various gaps when we talk about its protection and security shields. Lot of vulnerabilities have been recorded in the last decade in various devices ranging from a simple smart lock to some complex application like vehicles. Some of the main factor for this variability have been identified in the last few years like: absence of core control to control the flow of data, scale, a lot of attacking surfaces. Although the securities of the IoT is examined and considered inside the examination network. Hence a strategy was proposed which was ability-based access control to control the access to delicate information. However, the technique which was proposed create an unreasonable deferral which could possibly bargain the user's protection. Creators of used TLS and IPsec in order provide protection and verification, but the proposed technologies are very costly making it unfit for the use [3]. A protection the board method is proposed which estimates the danger of revealing information to other people, be that as it may, in reality, advantage of these IoT administrations top the danger of security disaster. There is how security mindful sharing of IoT information are required deprived of yielding protection of clients. In a nut cell these are the few works that had been done against the previously mentioned difficulties related to security and protection for IoT [4-6]. Every transaction received by the digger are checked by some approved marks which are contained inside the transaction. Each and every digger annexes the transaction which are pre checked to its very own pending blocks of transactions which are to be mined that are holed. The accompanying striking highpoints of Blockchain that make it more attractive of innovations inclined by the challenges in Internet of things.

- Decentralization: The nonattendance of focal control ensures versatility and power by using resources of each taking an intrigue center and taking out m-o (many-to-one) traffic streams, which hence reduces deferral and annihilations the issue of a lone reason for frustration.is appropriate for most IoT use situations where the character of the clients must be kept private [7].

- Anonymity: The innate secrecy oversaw is suitable for almost Internet of Things use circumstances and the character of the customer must be kept secret [2,4-6]. 
- Security: Blockchain comprehends an ensured framework which is appealing in Internet of things over untrusted parties with different and assorted devices [7].

Be that as it may, receiving BC in IoT isn't direct and will require tending to the accompanying basic difficulties: Mining is especially computationally concentrated, while most of IoT gadgets are asset limited. Even in most of the IoT applications small inertness is alluring, mining of blocks is tedious.

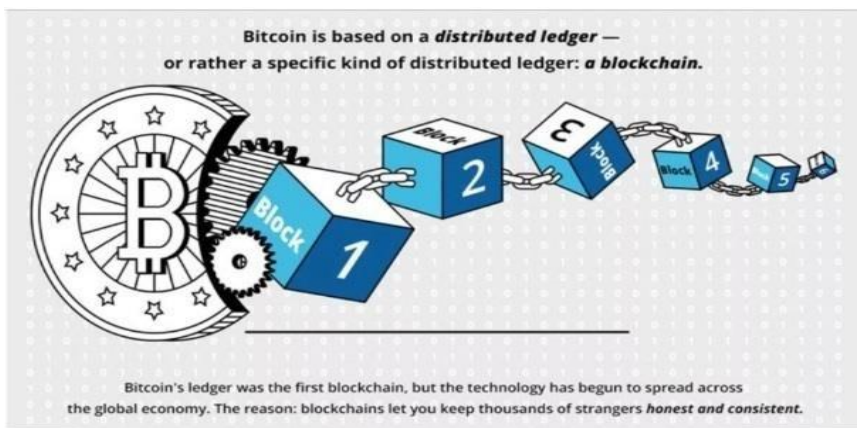

Fig.1. Bitcoin assembles blockchain [8]

This paper is categorized into seven section. Section 1, deals with introductory part of scope of blockchain in IoT, where as in section 2, is related work. Section 3 deals with need of blockchain in internet. In the next section $4 \& 54$ principle and strengths of blockchain and Use of blockchain in smart environment is discussed. In section 6 Challenging issues of blockchain are provided. In section 7 advantages and disadvantages are discussed. In the last conclusion of the work is discussed.

\section{RELATED WORK}

A. Reyna et.al. [1] discuss about the challenges to join the blockchain with the internet of things, the benefit of blockchain, possible topologies. S. Huckle et.al. [2] in his paper investigates how the Internet of Things and blockchain innovation can profit shared economy applications. The focal point of this examination is seeing how blockchain can be abused to make decentralized, shared economy applications that enable individuals to monetize, safely, their things to make more riches. A. Dorri et.al. [3] proposed another safe, private, and lightweight engineering for IoT, in view of BC innovation that disposes of the overhead of $\mathrm{BC}$ while keeping up the majority of its security and protection benefits. The Blockchain innovation initially came to unmistakable quality in mid-2009, through the cryptographic money Bitcoin (BTC) Bitcoin clients that are known by a variable Public Key (PK) create and communicate exchanges to the system to exchange cash. These exchanges are pushed into a square by clients. When a square is full, the square is affixed to the Blockchain by playing out a mining procedure. framework to guarantee the security and protection of data. J. Shen et.al., [4] applied shrewd contract in IoT. K. Christidis and M. Devetsikiotis, [5] utilized the BC stage to oversee IoT gadgets. S. Huh, S. Cho, and S. Kim [6] developed and discussed security transmission for IoT. C. Wang et.al., [7] explained the blast of intrigue with the Blockchain innovation and discussed applications that could recently run just through a believed middle person to work in a decentralized manner.

\section{NEED FOR BLOCKCHAIN}

Currently, The IoT represents about 5.0 billion associated gadgets, and even after this data will proceed to develop and achieve about 29.0 billion by the year 2022. Each gadget creates and trades information on the Internet. Therefore, thinking about these enormous number of gadgets, it is straightforward that we are discussing a broad and consistent creation of information. Tending to the principal of security problems for this tremendous data framework is a challenge within itself. By this segment, we try to examine the difficulties looked by some of the IoT organizations [9]. Basic challenge for Internet of things is knows as its dispersed engineering. Normally, in an Internet of things attacks, for example, DDoS (Distributed Denial-of-Service). [10] An arrangement of the hubs with a few tainted gadgets,acting all the while, can fall rapidly. Another worry respects it brought together design (commonly IoT condition influences on a focal cloud specialist co-op). arrange, every hub is conceivable purpose of its disappointment which can be abused to dispatch cyber Such an essential issue of disappointment is a weakness, which must be tended to. Another steady and presumably a standout amongst the most basic danger is information secrecy and confirmation. Without data security, IoT information can be abused and improperly utilized. What's more, with the development of new plans of action where gadgets can trade assets like information, computational power or power independently, information security ends up basic. Another test for Internet of things which is information integrity. The only critical utilizations of Internet of things is in the core decision supportive networks. The information amassed from armada of sensors which can be used to make decision timely. In this manner, framework from infusion attacks it is basic to shield, which attempt to infuse false event in addition along these lines, influence basic decision-making. [11] Accessibility is basic for computerized frameworks like vehicular systems, fabricating enterprises, and shrewd matrices which handle real time data. Sensor interruption can upshot in misfortunes differing starting financial toward perilous circumstances. By the rise of Mechanism Economy, whereby the sensors producing information exist equipped for exchanging information in information commercial centers and start to finish self-ruling framework, making trust between taking an interest substance is a critical test [12]. The nearness of an openly certain review trail without a believed outsider is attractive, in this way taking care of the issue of non-disavowal.

\section{PRINCIPLES AND STRENGTHS OF BLOCKCHAIN}

The essential objective of the Blockchain is to allow individuals from any type of trust we are presently compelled to provide for go-betweens who direct and "deal with" an extensive piece of natives' life.

Published By: 
The $\mathrm{BC}$ is an innovation that at first was utilized to advance business (exchanges) through another money which is autonomous of Bitcoin or we call it banks and States. This money is computerized, which is utilized in a form of method for trade acknowledged by the clients associated with an exchange. The strength of this cryptocurrency or all the more explicitly digital money is that there is no requirement for an open specialist. In any case, what is considerably more fascinating is to make sense of how to acknowledge and execute this new cryptographic money. To accomplish such an objective, a few advances, and cryptographic or security capacities which is misused. The collaboration within all these innovations comprises the Blockchain. In any case, this innovation is beginning are abused in few distinct settings and not only for Bitcoin. This is the most fascinating purpose of this progressive new innovation. Individuals regularly mistake $\mathrm{BC}$ for Bitcoin; however, Bitcoin demonstrates a cryptographic money that use the $\mathrm{BC}$ innovation to most likely unreservedly and internationally circle without the supervision of a focal underwriter (the banks)
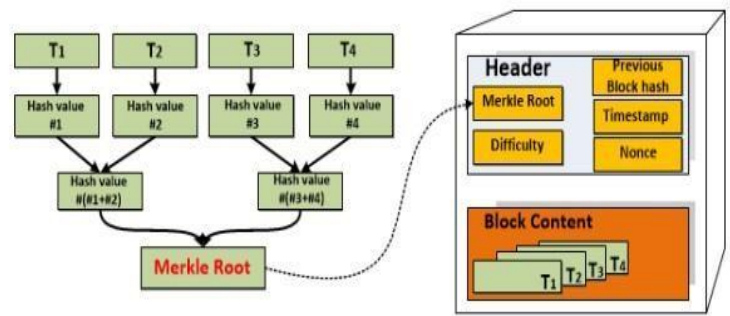

Merkle Root $=a[(4(41+n 2)+t(43+n 4)]$ $\mathrm{T}_{\mathrm{n}}=$ Transaction $\mathrm{n}$

Fig.2. Block Structure [13]

The essential objective of the Blockchain is to allow individuals from any type of trust we are presently compelled to provide for go-betweens who direct and "deal with" an extensive piece of natives' life. The $\mathrm{BC}$ is an innovation that at first was utilized to advance business (exchanges) through another money which is autonomous of Bitcoin or we call it banks and States. This money is computerized, which is utilized in a form of method for trade acknowledged by the clients associated with an exchange. The strength of this cryptocurrency or all the more explicitly digital money is that there is no requirement for an open specialist. In any case, what is considerably more fascinating is to make sense of how to acknowledge and execute this new cryptographic money. To accomplish such an objective, a few advances, and cryptographic or security capacities which is misused. The collaboration within all these innovations comprises the Blockchain. In any case, this innovation is beginning are abused in few distinct settings and not only for Bitcoin. This is the most fascinating purpose of this progressive new innovation. Individuals regularly mistake BC for Bitcoin; however, Bitcoin demonstrates a cryptographic money that use the $\mathrm{BC}$ innovation to most likely unreservedly and internationally circle without the supervision of a focal underwriter (the banks).

At the end of the day, Bitcoin or cryptocurrency is just budgetary which make utilization of such a incredible innovation. Formerly we plunge into the Blockchain innovation \& make sense of what Blockchain endeavors to comprehend, it is critical to make a vital prerequisite. The Blockchain is only a conveyed databank framework dependent in accord decides permit the exchange of significant worth among substances.

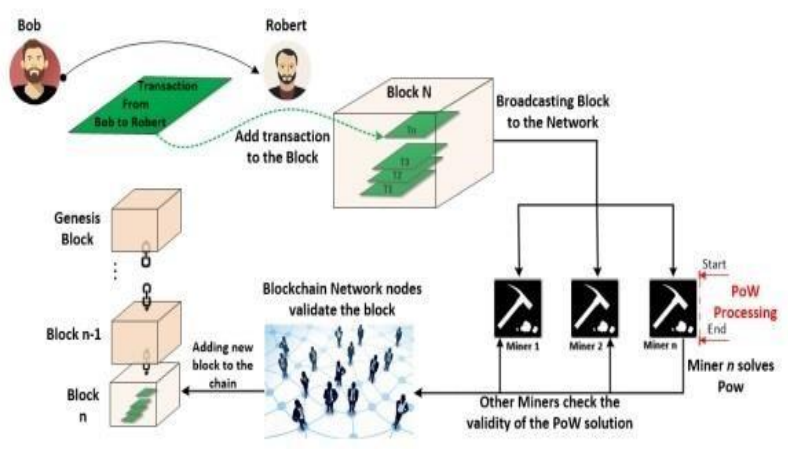

Fig.3. Transaction block validation and addition flow [14]

At hand are numerous appropriated frameworks dependent in agreement calculations, however the Blockchain is one of the special cases which at same time appreciates the accompanying three properties:

O Trustless: There is no compelling reason to claim an ensured computerized personality. The included elements don't have any acquaintance with one another, yet they can at any rate trade information without knowing their individual personalities [15].

O Permission less: Nobody chooses who can or cannot work on the BC arrange. There are neither. authorizations nor controllers [16].

O Oversight safe: Blockchain presence a system lacking regulators, where substances trust is just a nature of the cryptographic calculations which administer any activity, anybody could execute the Blockchain [17].

Any transaction, when acknowledged, cannot be situated halted or else blue-penciled. Furthermore, Blockchain can be classified into two dependents in its working: permission less. Then again, permission less $\mathrm{BC}$ enables anybody to join the system, take part during the time spent square confirmation to achieve an accord and furthermore make shrewd contracts. After this basic refinement, it is basic to comprehend what $\mathrm{BC}$ endeavors to settle. How about we think about the conventional method to make monetary transactions among people. How about we expect that Harry needs to send cash to Jack. To ensure this, a brought together TTP (Trusted Third Party) needs to play out the accompanying three stages:

(I) Know Jack's state (e.g., India),

(II) Know Jack's financial balance, \& 
(III) Make transaction from harry to jack. Misusing Blockchain, it is conceivable to acquire a similar outcome however by more or less progress by evacuating Trusted Third Party (TTP), creating the transaction quicker less than 3days: prompt, and creating the transaction less expensive: Bitcoin transaction charges are deliberate.

Blockchain innovation depends on 4 focal ideas:

(I) A distributed system: this arrangement expels the focal TTP inferring all hubs inside the system have similar benefits. In this system, hubs can cooperate with one another using a couple of private/open keys. Private key is known for sign transaction open key is for location determination on the system [18].

(II) Open \& circulated record: Envision any record as a bookkeeping book gathering every one of the transactions of the system in sequential request. This information structure is definitely not a brought together substance, however every hub has got its own duplicate of it. The record is open and open to everybody. Everybody on the system can see where the advantage is and how much resource everyone has in his/her record also. Every hub can see whether a transaction is legitimate or not [19].

(III) Synchronization of Ledger copies: In this situation, where hubs have their very own duplicate of a similar record, an approach to synchronize records crosswise over hubs is required. To achieve such an objective, three fundamental advances are required [20].

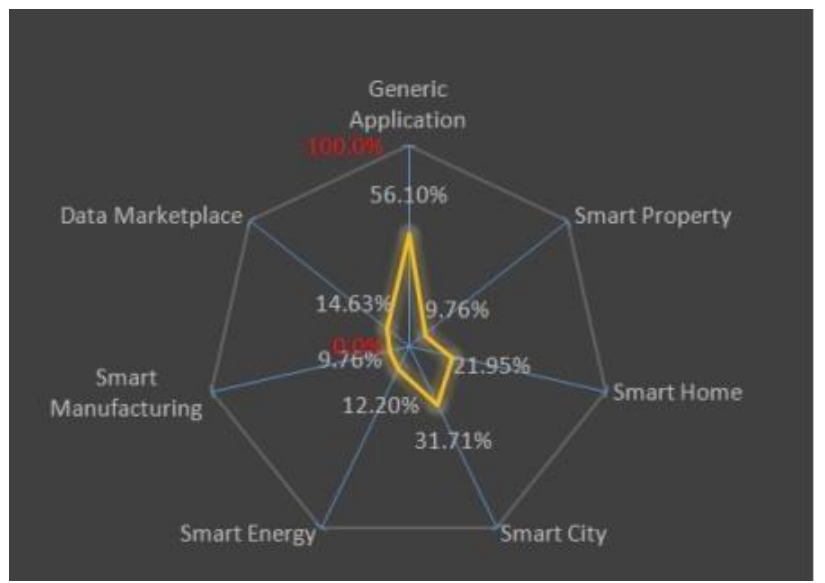

Fig.4. Paper scope distribution [21]

We can add transaction to the chain using one kind of hub known as miners will contend among themselves to comprehend will's identity the first to take the new transaction, approve it and place it into record. The miner will earn money after doing that. At start miner has to approve the transaction and to illuminate a scientific speculating amusement. Along these lines, just a single mine worker adds transactions to the Blockchain at any time. Additionally, to maintain a strategic distance from assaults to the framework like the outstanding "double spending assault", an answer for make the "amusement" hard for untrustworthy miners is required. This arrangement is basically to make it costly (contribute a great deal of PC handling control) for enemies to include transactions.

(IV) In this way, in basic words, each interval a gathering transaction is affirmed, that is associated with past block through a hash, a one of a kind and permanent stamp that gives the certification that nobody can alter the recorded information. The best way to alter the $\mathrm{BC}$ is to pick up the $51 \%$ of the processing intensity of the entire system following up on the BC. In this manner, which is outlandish for a person to make modifications to the record. That is a crucial component: the devolution of the Blockchain is the thing that makes it safe \& disseminated; besides, such a devolution permits the disposal of any focal element, depending rather on "democracy of computing power," guaranteed via a large number of members in Blockchain [22].

\section{USE OF BLOCKCHAIN IN SMART ENVIRONMENTS}

As we would like to think, BC speaks to the missing bit of a riddle to unravel protection and dependability imperfections in Internet of Things. The natural distributed, independent, \& trustless highlights of Blockchain make it reasonable get connected in just few unique situations, for example, "Smart Home," "Smart Industries," and "Smart Grid," also. For instance, the Blockchain can keep a changeless past of keen gadgets. In addition, it might empower a self-sufficient working of keen gadgets, evacuating the nearness of incorporated expert or hominoid governor by the utilization of savvy agreements. Besides, Blockchain can likewise make the protected path for savvy gadgets trading messages with one another. Accordingly, the objective of paper is being to make sense of how Blockchain can encounter the Internet of things security \& protection prerequisites or all in all how Blockchain can be coordinated with Internet of things. In this way, in this area, we will order the examined papers in four primary gatherings, considering the field where everyone works. The measured gatherings are

1. Smart City and 2. Generic Context.

The Smart City is additionally classified hooked on two sub-sets:

\section{Smart Industry}

\section{Smart Grid.}

Table-1 speaks to this classification. Above Table orders the overviewed arrangement into two classes

1. data manipulation 2. device manipulation.

An information control style uses the Blockchain as protected archive abusing its highlights like a permanent open record and capacity to make an advanced trail for confirmation. A gadget control approach, then again, uses BC as a safe book of records, yet uses shrewd contracts to make self-sufficient frameworks equipped for settling on choices based on business rationale. Savvy contracts likewise wipe out the requirement for a believed outsider as the guidelines are executed naturally dependent on current conditions and standards are freely accessible in this manner advancing straightforwardness.

Published By: 
Studies shows that blockchain can be used in our day to day life in various ways making the smart use of our internet, some of the application of blockchain in internet of things or commonly knows as blockchain of things is shown in below figure:

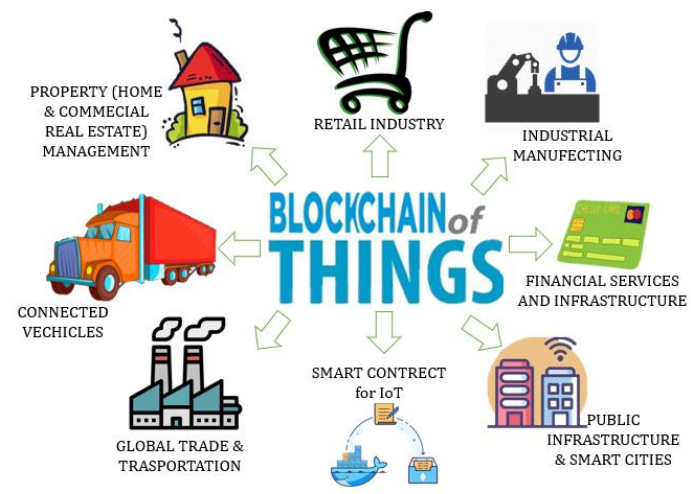

Fig.5. Application of Blockchain

\section{CHALLENGING ISSUES OF BLOCK CHAIN}

An IoT biological system has various vulnerabilities concerning secrecy, security, and data integrity. Therefore, the scientists and engineers of the ICT division chose to incorporate "security by plan" innovation inside a domain to such an extent that Internet of things beats constraints. Blockchain, one of such innovation, awards genuineness, non-disavowal, \& integrity of course, plus using savvy contracts, oversees approval and mechanization of exchanges also [23]. In this paper we,

$>$ thought about various application regions, sorting out the accessible writing in these fields,

$>$ exhibited two utilization designs that are: gadget control and data the board (open commercial center arrangement), and

$>$ examined the advancement dimension of some of the introduced arrangements.

As we would like to think, there are numerous potential outcomes for future research ways. We trust that, in a period portrayed by the unavoidable utilization of shrewd gadgets and generation of the gigantic measure of (Big data) data the essential two needs are:

$>$ Advancement of an answer for allow data protection and integrity; and

$>$ Plan of framework ready to deal with the extraordinary character of gadgets in a carefully designed way

\begin{tabular}{|c|c|c|}
\hline Papers & Device Manipulation & Data Manipulation \\
\hline TransActive Grid & - & d \\
\hline Filament & - & 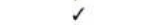 \\
\hline Jentzsch et al. (Slock.it) & $\checkmark$ & . \\
\hline Zhumabekuly Aitzhan et al. (PriWatt) & . & $\checkmark$ \\
\hline Axon et al. & $s$ & - \\
\hline Bahga et al. & $\checkmark$ & - \\
\hline Biswas et al. & . & 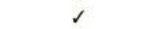 \\
\hline Brody (ADEPT) & $\checkmark$ & . \\
\hline Cha et al. & $s$ & 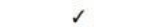 \\
\hline Conoscents et al. & - & $s$ \\
\hline Dorri et al. & & 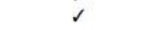 \\
\hline Ghuli et al. & $s$ & - \\
\hline Hardjono et al & $s$ & 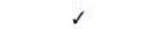 \\
\hline Hashemi et al. & : & $\checkmark$ \\
\hline Herbert et al. & $\checkmark$ & . \\
\hline Huhet al. & $\checkmark$ & - \\
\hline Hyper-Fabric & : & $\checkmark$ \\
\hline Leiding et al. & . & : \\
\hline Lombardi et al. & $i$ & : \\
\hline Munsing et al. & $s$ & - \\
\hline Nehai et al. & . & 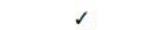 \\
\hline Ouaddah et al. & $\checkmark$ & 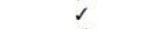 \\
\hline Prabhu et al. & $s$ & $s$ \\
\hline Shafagh et al & . & $s$ \\
\hline $\begin{array}{l}\text { Snatagnet ai } \\
\text { Sikorski et al. }\end{array}$ & : & 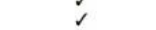 \\
\hline Wilkinson et al. (Stori) & $\checkmark$ & 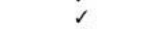 \\
\hline $\begin{array}{l}\text { Wörner et al. } \\
\text {. }\end{array}$ & $\checkmark$ & 6 \\
\hline Zhang et al. & : & 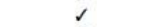 \\
\hline Zyskind et al. & . & 1 \\
\hline Xu et al. (Sapphire) & 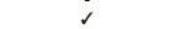 & . \\
\hline Ralph Deters & . & 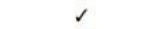 \\
\hline Gorilovsky et al. (Moeco) & . & 6 \\
\hline
\end{tabular}

Table 1. Usage pattern Organization [24]

\section{ADVANTAGES AND DISADVANTAGES}

There are several advantages and disadvantages of Block chain in IoT are present. Some of the advantages are listed below:

- No Fraud: As blockchain makes record open source. We can see any transaction form any block so there is no chance of any extortion occurrence [25].

- Government has no Interference: No organization in our county has any control over this virtual money so as a result there is no interference by any administration [26].

- Instant Transactions: It is being observed that the transaction done via blockchain technologies is $10 \mathrm{x}$ faster than the traditional bank one [27].

- Financial Efficiency is improved: The blockchain make it possible to exclude the middle ones in making any transaction because it is a straightforward transaction no outsider is required in this transaction [28].

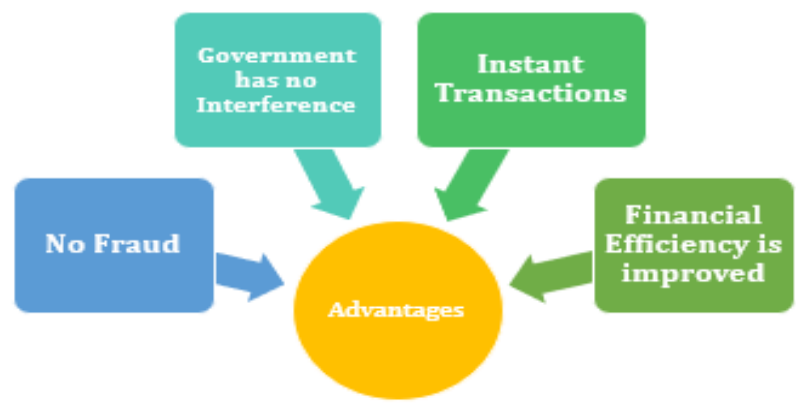

Fig.6. Advantages of Blockchain

Some of the major disadvantages of Block chain in IoT are presented below:

- Extremely Volatile in nature: The standard use un blockchain innovation are exposed to unpredictability. For example, Bitcoin which change it value each and every day [29].

- Crime: As it is a virtual in form of decentralized blockchain standards which create unlawful exchanges, a basic crime [30]. 


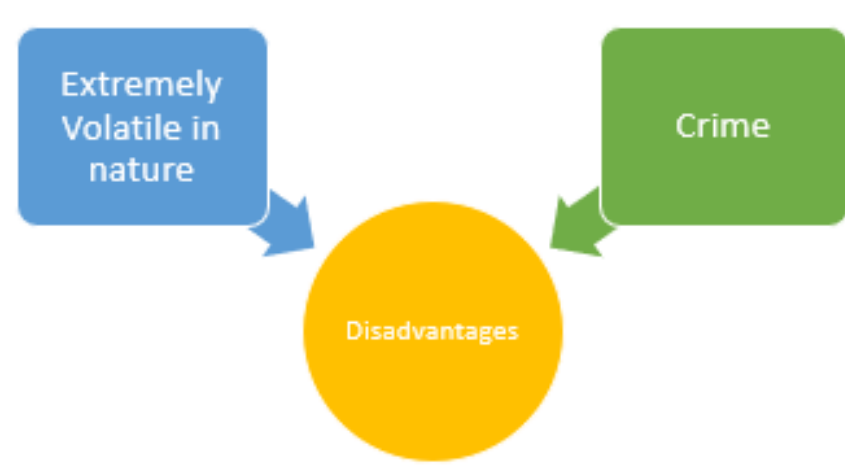

Fig.7. Disadvantages of Blockchain

\section{CONCLUSION}

This paper has helped us in analyzing the main challenges of blockchain and IoT that must be address to make them work efficiently together. Blockchain contain the capability to change the entire internet by presenting new standard of data transparency and peer to peer communication. It can create a market worth trillion in near feature. The field of IoT and blockchain together create a vision of development, it has an ability to create internet with secure and equal framework for interaction, where no one is in charge. Blockchain can possibly bolster our everyday life dependent on machine to machine interaction. In near future we won't be limited to only AI interaction, for example a smart refrigerator will automatically order the apples with virtual currency, it can even negotiate the price taking the owner preference.

\section{REFERENCES}

1. Ana Reyna, Cristian Martín, Jaime Chen, Enrique Soler and Manuel Díaz "On blockchain and its integration with IoT. Challenges and opportunities" Volume 88, November 2018

2. Internet of Things, Blockchain and Shared Economy Applications by Steve Huckle, Rituparna Bhattacharya, Martin White and Natalia Beloff ,Volume 98, 2016, Pages 461-466

3. Ali Dorri, Salil S. Kanhere, and Raja Jurdak "Blockchain in Internet of Things: Challenges and Solutions"

4. J. Shen et.al., "Secure data uploading scheme for a smart home system," Information Sciences, vol. 453, pp. 186-197, 2018.

5. K. Christidis and M. Devetsikiotis, "Blockchain and Smart Contracts for the Internet of Things," IEEE Access, vol. 4, pp. 2292-2303, 2016.

6. S. Huh, S. Cho, and S. Kim, "Managing IoT devices using blockchain platform," in Proceedings of the 19th International Conference on Advanced Communications Technology, ICACT 2017, pp. 464-467, kor, February 2017.

7. C. Wang et.al., "A Novel Security Scheme Based on Instant Encrypted Transmission for Internet of Things," Security and Communication Networks, vol. 2018, pp. 1-7, 2018

8. Bitcoin assembles blockchain. Available at: https: www.investopedia.com/terms/b/blockchain.asp [accessed on 10 march 2019]

9. Barboutov, K.; Furuskär, A.; Inam, R.; Lindberg, P.; Öhman, K.; Sachs, J.; Sveningsson, R.; Torsner, J.; Wallstedt, K. Ericsson Mobility Report. [accessed on 10 March 2019].

10. https://www2.deloitte.com/de/de/pages/technology-media-and-telecom munications/articles/cyber-security-prevention-of-ddos-attacks-with-bl ockchain-technology.html [accessed on 11-march-2019]

11. Gubbi, J.; Buyya, R.; Marusic, S.; Palaniswami, M. Internet of Things (IoT): A vision, architectural elements, and future directions. Future Gener. Comput. Syst. 2013, 29, 1645-1660.

12. Kolias, C.; Kambourakis, G.; Stavrou, A.; Voas, J. DDoS in the IoT: Mirai and other botnets. Comput. 2017, 50, 80-84.

13. Block Structure. Available at: 12-March-2019]
14. Transaction block validation and addition flow. Available at: http://www.reseachgate.net/publication/326868072 [accessed on 19-March-2019]

15. Trustless Available

at: https://lisk.io/academy/blockchain-basics/benefits-of-blockchain/why-i s-blockchain-trustless [accessed on 10-march-2019]

16. Permission less Available https://www blockchain-councilorg/blockchain/advantages-and-di antages-of-permissionless-blockchain/ [accessed on 10-march-2019]

17. Oversight safe Available at: https://cryptodisrupt.com/blockchain-oversight-could-change-arms-tra $\mathrm{de} /$ [accessed on 10-march-2019]

18. Sicari, S.; Rizzardi, A.; Cappiello, C.; Miorandi, D.; Coen-Porisini, A. Toward data governance in the internet of things. In New Advances in the Internet of Things; Springer:Cham, Germany, 2018;pp. 59-74

19. Open \& circulated record ,Available at: https://hackernoon.com/blockchain-technology-explained-introduction -meaning-and-applications-edbd6759a2b2 [accessed on 10 march-2019]

20. Synchronization of Ledger copies, Available at :https://codeburst.io/distributed-ledger-technology-fundamentals-youmust-know-2d0f82628258 [accessed on 10-march-2019] Paper scope distribution. Available at: http://www.reseachgate.net/publication/326868072 [accessed on 10 March 2019]

21. Crucial component, Available at https://medium.com/permissionio/democracy-on-the-blockchain-6bb3 7c2e893f [accessed on 11-march-2019]

22. Challenging Issues Of Blockchain, Available at https://www.coindesk.com/information/blockchains-issues-limitations [accessed on 11-march-2019]

23. Usage pattern Organization available at http://www.reseachgate.net/publication/326868072 [accessed on 10 March 2019]

24. https://www.ibm.com/blogs/blockchain/2017/09/three-features-of-bloc kchain-that-help-prevent-fraud/ [accessed on 12-march-2019]

25. https://medium.com/coinmonks/blockchain-is-self-regulation-sufficien $\mathrm{t}-5 \mathrm{bb} 68 \mathrm{ac} 7 \mathrm{e} 33 \mathrm{f}$ [accessed on 12-march-2019]

26. https://www.quora.com/Is-a-Bitcoin-transaction-truly-instant [accessed on 12-march-2019]

27. https://www.coindesk.com/blockchain-aid-efficiency [accessed on 12-march-2019]

28. https://www.investopedia.com/articles/investing/052014/why-bitcoinsvalue-so-volatile.asp [accessed on 13-march-2019]

29. http://www.police-foundation.org.uk/2018/09/is-blockchain-good-new s-or-bad-when-it-comes-to-policing-and-crime/ [accessed on 13-march-2019]

30. TIAGO M. FERNÁNDEZ-CARAMÉS, (Senior Member, IEEE), and PAULA FRAGA-LAMAS, (Member, IEEE) "A Review on the Use of Blockchain for the Internet of Things" Department of Computer Engineering, Faculty of Computer Science, Campus de Elviña, s/n, Universidade da Coruña, April 11, 2018.

31. Dr. Xing Liu,Kwantlen Polytechnic University,Surrey, B.C., CANADA "Internet of Things Based on Blockchain" IEEE IEMCON 2018 - The 9 th IEEE Annual Information Technology, Electronics \& Mobile Communication Conference 1-3 November, 2018

32. Francesco Restuccia, Member, IEEE, Salvatore D'Oro, Member, IEEE, Salil S. Kanhere, Senior Member, IEEE, Tommaso Melodia, Fellow, IEEE, and Sajal K. Das, Fellow, IEEE "Blockchain for the Internet of Things: Present and IFuture" IEEE INTERNET OF THINGS JOURNAL, VOL. 1, NO. 1, JANUARY 2018

33. D.Salma Faroze Department of Computer Science and Engineering CBIT College Proddatur, And Pallavolu Andhra Pradesh - India "Block Chain \& Internet of Things: Security, Challenges, Research Issues" International Journal of Computer Science Trends and Technology (IJCST) - Volume 6 Issue 5, Sep-Oct 2018

34. L. Sharma, N.Lohan, “ Internet of Things with Object detection: Challenges, Applications, and Solutions", Handbook of Research on Big Data and the IoT, IGI Global, pp. 89-100, March 2019.

35. L. Sharma, D. Yadav, A. Singh, "Fisher's linear discriminant ratio-based threshold for moving human detection in thermal video", Infrared Physics \& Technology, Elsevier, vol. 78, pp. 118-128, Sept. 2016. 
36. L. Sharma, D. Yadav, "Histogram-based adaptive learning for background modelling: moving object detection in video surveillance", International Journal of Telemedicine and Clinical Practices, Inderscience, vol. 2, no. 1, pp. 74-92, 2017.

37. L.Sharma, Nirvikar Lohan, "Performance analysis of moving object detection using BGS techniques in visual surveillance", International Journal of SpatioTemporal Data Science, Vol.1 No.1, pp.22 - 53, Jan. 2019

\section{AUTHORS PROFILE}

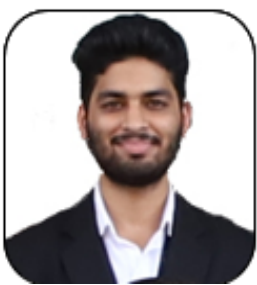

Anubhav Kumar is an BCA graduate in the field of information technology form Amity University. $\mathrm{He}$ is a recipient of several prestigious awards during academic career. He had worked in Airports Authority of India Safdarjung, Delhi to apply his strategic analytical skills in the field of Network Infrastructure. He has adequate knowledge of different computer related programming language. His current research interests include cyber security, AI and blockchain and Internet of Things.

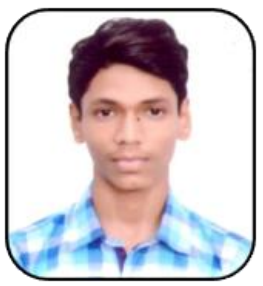

Gaurav Jha is a BCA graduate student in the feild of information technology. He had worked in KR Network cloud Technologies Pvt. Limited, Delhi. He also developed an Android application named chat-bot. And he has great knowledge in the field of cyber security. His current research interests include cyber security, AI and blockchain.

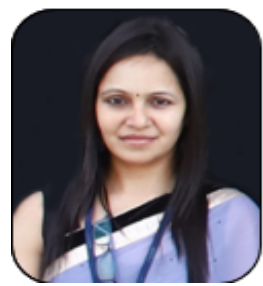

Dr. Lavanya Sharma is a Ph.D. graduate in the domain of Computer Vision at Uttarakhand Technical University (govt.), India and an assistant professor, Amity Institute of Information Technology at Amity University UP, Noida, India. She is an active nationally recognized researcher who produces dozens of papers in her field. She is an Editorial Member/ Reviewer of various journal of repute and active program committee member of various IEEE and Springer conferences also She is an active nationally recognized computer vision educator who has produced dozens of papers

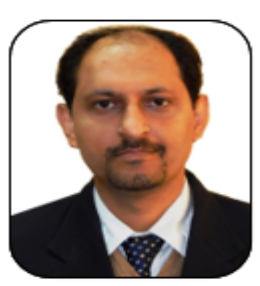

Prof. (Dr.) Sunil Kumar Khatri is Director, Amity Institute of Information Technology and Additional Director, Amity Directorate of Engineering \& Technology at Amity University UP, Noida, India. He is a Fellow of IETE, Sr. Life Member of CSI, IEEE, IASCSIT and Member of IAENG. He is Secretary in SREQOM, Convener, EAC, IEEE UP Section Executive Council and Past Vice-Chairman of CSI Noida Chapter. Dr. Sunil Kumar Khatri is Editor IJSAEM, Springer Verlag. He is in Editorial Board of several Journals from USA, Egypt, Hong Kong, Singapore and India. He has eleven edited books, nine guest edited special issues of international journals, eleven patents filed and more than 125 papers in international and national journals and proceedings. His areas of research are Artificial Intelligence, Software Reliability and Testing, and Data Analytics. 